\title{
Stability of Various Types of Aspheric Intraocular Lenses After Implantation: A One-Year Retrospective Study
}

\author{
Yuan Ning' \\ Yushuang Shao' \\ Jiangyue Zhao' \\ Jinsong Zhang' \\ Mingwu Wang ${ }^{2}$ \\ Yu Qin'
}

'Department of Ophthalmology, The Fourth Affiliated Hospital of China Medical University, Eye Hospital of China Medical University, Key Lens Research Laboratory of Liaoning Province, Shenyang, I 10005, People's Republic of China; ${ }^{2}$ Department of Ophthalmology and Vision Science, The University of Arizona College of Medicine, Tucson, Arizona, 857|I-1824, USA
Correspondence: Yu Qin

Department of Ophthalmology, The Fourth Affiliated Hospital of China Medical University, Eye Hospital of China Medical University, Key Lens Research Laboratory of Liaoning Province, No. II Xinhua Road, Heping District, Shenyang, I 10005, People's Republic of China

Tel +8624620350I5

Fax +862462035015

Email yuqin_3620@I63.com
Objective: The aim of the present study was to evaluate the stability of four different types of aspheric intraocular lenses (IOLs) after implantation.

Methods: This retrospective study included 124 eyes implanted with four different types of aspheric IOLs including a one-piece four-loop fixed hydrophilic acrylic IOL, a one-piece flat fixed acrylic IOL, a one-piece two-loop fixed acrylic IOL, and a three-piece two-loop fixed silicone IOL. IOL decentration, tilt, and ocular coma-like aberration (coma) at one-week, one-month, three-month, and one-year time points were evaluated postoperatively.

Results: IOL decentration, tilt, and coma in the AO, 36A, IQ, and KS-AiN implantation groups were statistically significantly different one week and one year postoperatively $(p<$ $0.01)$. There were significant differences between each of the pairs of groups $(p<0.01)$, except for coma between the AO and 36A implantation groups one week postoperatively $(p>0.05)$. When comparing the different time points (ie, one week, one month, three months, and one year postoperatively), IOL decentration, tilt, and coma were significantly different in each group $(p<0.05)$. IOL decentration, tilt, and coma in each group increased over the period from one week to one year postoperatively. A positive linear correlation was observed between IOL decentration or tilt and coma one year postoperatively $(p<0.01)$.

Conclusion: One-piece multi-point fixed acrylic IOLs demonstrate better stability when compared with three-piece two-point fixed silicone IOLs. IOL decentration, tilt, and coma increase gradually over time. Ocular coma-like aberrations are influenced by the stability of IOLs.

Keywords: stability, aspheric intraocular lens, IOL, decentration, tilt, coma

\section{Introduction}

With the advancement in surgical techniques, cataract surgery has evolved into a type of refractive surgery. The success rate of intraocular lens (IOL) in-the-bag implantation has increased greatly, while the rate of serious IOL dislocation has decreased significantly. However, IOL tilt and decentration are still inevitable. ${ }^{1}$ It has been reported that the incidence of abnormal IOL positioning, which is one of the most common postoperative complications, ranges from $7 \%$ to $40 \%{ }^{2}$ One study showed that the asymmetry of the fixed-loop model (capsular bag-ciliary sulcus fixation) is one of the most common causes of abnormal IOL positioning. ${ }^{3}$ The stability of IOLs is mainly reflected by decentration and tilt. ${ }^{4,5}$ Serious IOL decentration and tilt may cause symptoms including loss of visual acuity, glare, halos, and mono diplopia. ${ }^{6}$ Even in patients with good visual acuity, slight 
decentration and tilt may occur. ${ }^{7}$ It has been shown that the visual quality of aspheric IOLs may decline to a greater degree than that of spherical IOLs if tilt is greater than $10^{\circ}$ and decentration is greater than $0.8 \mathrm{~mm} .{ }^{8}$ Decentration and tilt play a critical role in the evaluation of visual quality after IOL implantation.

It has been demonstrated that aspheric IOLs possess obvious advantages in reducing wavefront aberrations and improving visual quality. ${ }^{9,10}$ Therefore, aspheric IOLs have recently been widely used clinically. The stability of various types of aspheric IOLs is different depending on the loop design, the material composition of the optical element, and the histocompatibility of the IOL. Ocular high-order wavefront aberrations, especially ocular comalike aberrations (coma), are affected by various types of aspheric IOLs. ${ }^{11}$

The aim of the present study is to compare decentration, tilt, and coma after implantation of four types of aspheric IOLs and to further investigate the stability of the different types of aspheric IOLs at various time points.

\section{Materials and Methods}

This retrospective study consisted of 124 eyes from 89 patients with age-related cataracts from June 2017 to June 2019 in the Department of Ophthalmology at the Fourth Affiliated Hospital of the China Medical University. The study protocol was approved by the institutional review board of the hospital, and the study was conducted in accordance with the 1964 Declaration of Helsinki and its later amendments. All patients provided written informed consent prior to study recruitment and enrollment.

Patients with age-related cataracts aged between 60 and 80 years with an axial length of between 22.0 and $24.0 \mathrm{~mm}$, preoperative corneal astigmatism of $<1.0$ diopter, nuclear hardness of grade II to IV based on the Emery-Little classification, and a corneal endothelial cell count of $>2000$ cells/ $\mathrm{mm}^{2}$ were selected. Exclusion criteria included other eye diseases such as glaucoma, uveitis, vitreoretinopathy, and high myopia; a history of intraocular surgery; after YAG laser surgery; intraoperative posterior capsular rupture; nonin-the-bag IOL implantation; excessive postoperative capsular fibrosis and anterior capsule shrinkage syndrome; postoperative IOL capsular capture; discoria and pupil displacement; and failure to follow up. Phacoemulsification and IOL implantation were performed by the same skilled surgeon through a $3.0 \mathrm{~mm}$ clear corneal incision.

Patients' demographics are shown in Table 1, and no statistically significant differences were detected between
Table I Characteristics of 89 Patients with Four Types of Aspheric IOLs Implantation

\begin{tabular}{|l|l|l|l|l|l|}
\hline \multirow{2}{*}{ Characteristic } & \multicolumn{4}{|l|}{ Mean \pm SD } & \multirow{2}{*}{ P-value } \\
\cline { 2 - 5 } & AO & 36A & IQ & $\begin{array}{l}\text { KS- } \\
\text { AiN }\end{array}$ & \\
\hline No. of patients & 24 & 22 & 21 & 22 & - \\
\hline No. of eyes (n) & 32 & 31 & 30 & 31 & - \\
\hline Age (y) & $\begin{array}{l}69.25 \\
\pm 5.86\end{array}$ & $\begin{array}{l}68.97 \\
\pm 6.12\end{array}$ & $\begin{array}{l}69.16 \\
\pm 6.08\end{array}$ & $\begin{array}{l}68.69 \\
\pm 6.32\end{array}$ & $0.826^{+}$ \\
\hline $\begin{array}{l}\text { Sex (male/ } \\
\text { female) }\end{array}$ & $11 / 13$ & $12 / 10$ & $11 / 10$ & $12 / 10$ & $0.763^{++}$ \\
\hline $\begin{array}{l}\text { Axial length } \\
\text { (mm) }\end{array}$ & $\begin{array}{l}23.02 \\
\pm 0.87\end{array}$ & $\begin{array}{l}22.95 \\
\pm 1.01\end{array}$ & $\begin{array}{l}22.86 \\
\pm 0.92\end{array}$ & $\begin{array}{l}23.12 \\
\pm 0.98\end{array}$ & $0.387^{+}$ \\
\hline Pupil size (mm) & $\begin{array}{l}2.86 \\
\pm 0.82\end{array}$ & $\begin{array}{l}2.92 \\
\pm 0.76\end{array}$ & $\begin{array}{l}3.06 \\
\pm 0.71\end{array}$ & $\begin{array}{l}2.98 \\
\pm 0.85\end{array}$ & $0.548^{+}$ \\
\hline
\end{tabular}

Notes: Data are mean \pm standard deviation unless otherwise indicated. ${ }^{+}$One-way ANOVA. ${ }^{++}$Chi-square tests.

the groups in terms of age, sex, axial length, or pupil size $(p>0.05)$.

\section{Intraocular Lens Selection}

Four different types of aspheric IOLs were selected including a one-piece four-loop fixed hydrophilic acrylic IOL (Akreos Advanced Optics, Bausch \& Lomb; AO), a onepiece flat fixed acrylic IOL (Acri. Smart 36A, Zeiss, Germany; 36A), a one-piece two-loop fixed acrylic IOL (AcrySof IQ SN60WF, Alcon; IQ), and a three-piece twoloop fixed silicone IOL (Canon Staar, Japan; KS-AiN), the characteristics of which are shown in Table 2.

\section{Outcome Measurements}

In this study, IOL decentration and tilt were examined using the Pentacam ${ }^{\circledR}$ HR Scheimpflug system (OCULUS, Wetzlar, Germany). Pupils were dilated to $8 \mathrm{~mm}$ before examination with $1 \%$ compound tropicamide eye drops (see Figure 1A). Coma was measured using the iTrace aberrometer (Tracey Technologies, Houston, TX, USA). Pupils were not dilated before the examination, and the diameter was set to $4 \mathrm{~mm}$ (see Figure 1B). Evaluations were performed one week, one month, three months, and one year postoperatively.

Scheimpflug cross-sectional images at $0^{\circ}-180^{\circ}$, $45^{\circ}-225^{\circ}, 90^{\circ}-270^{\circ}$, and $135^{\circ}-315^{\circ}$ in the target eye were selected for measurement. IOL tilt and 


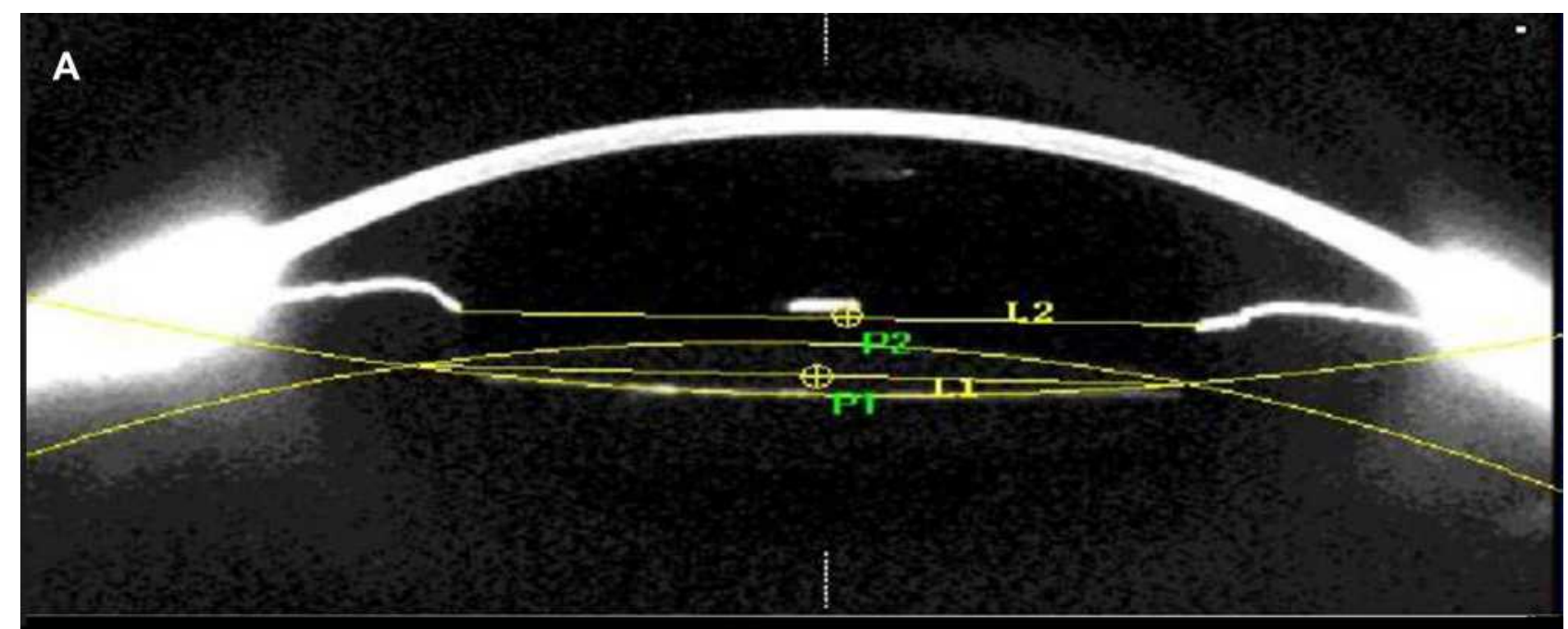

B

WF Summary Display

\section{iTrace , maser}

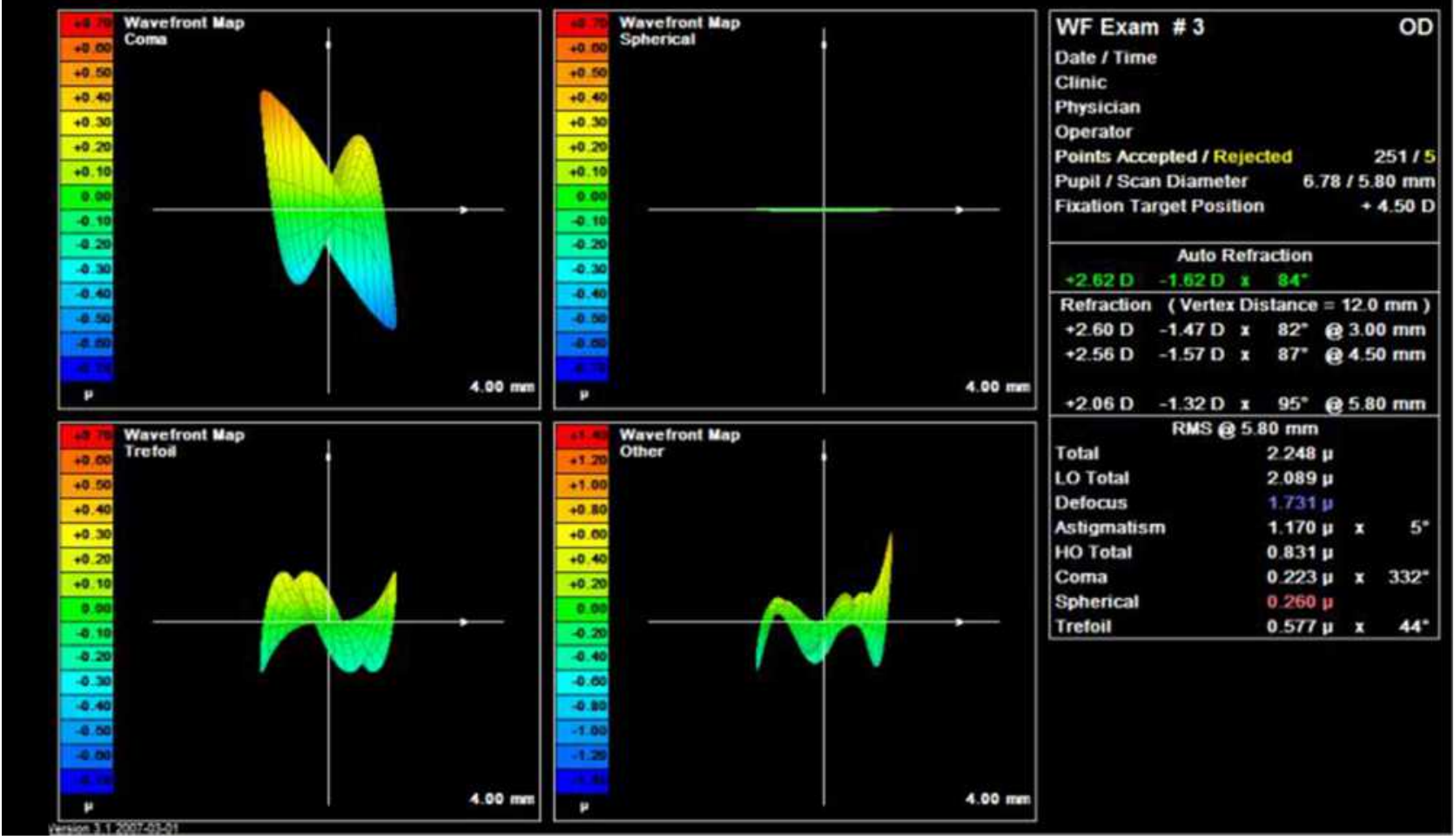

Figure I (A) IOL decentration and tilt were examined using the Pentacam ${ }^{\circledR}$ HR Scheimpflug system (Oculus, Wetzlar, Germany). (B) Coma was measured using the iTrace aberrometer (Tracey Technologies, Houston, TX, USA).

decentration in the cross-sectional images were calculated using Image-Pro Plus version 6.0 professional image analysis software. The pupil center was estimated as the midpoint of the two visible pupil segments, while the IOL center was the midpoint of the intersection of the two spheres fitting the anterior and posterior edges of the IOL, and the IOL was the line joining the centers of the curvature of the anterior and posterior surfaces of the IOL. Decentration was measured from the center of the IOL to the pupillary axis, which was perpendicular to line L2 (see Figure 1A). Tilt was measured as the angle between the IOL axis, 
Table 2 Characteristics of Four Different Types of Aspheric IOLs

\begin{tabular}{|l|c|c|c|c|c|c|c|c|c|}
\hline IOL & $\begin{array}{c}\text { No. } \\
\text { of } \\
\text { Eyes } \\
(\mathbf{n})\end{array}$ & Manufacturer & Model & Design & $\begin{array}{c}\text { Optical } \\
\text { Materials }\end{array}$ & $\begin{array}{c}\text { Loop } \\
\text { Design }\end{array}$ & $\begin{array}{c}\text { Spherical } \\
\text { Aberration ( } \boldsymbol{\mu m})\end{array}$ & $\begin{array}{c}\text { Loop } \\
\text { Angle }\end{array}$ & $\begin{array}{c}\text { Optical } \\
\text { Diameter (mm) }\end{array}$ \\
\hline AO & 32 & Bausch \& Lomb & $\begin{array}{c}\text { Akreos } \\
\text { AO }\end{array}$ & $\begin{array}{c}\text { One- } \\
\text { piece }\end{array}$ & Acrylate & 4 & 0 & 0 & 6.0 \\
\hline $36 \mathrm{~A}$ & 31 & Zeiss Germany & $\begin{array}{c}\text { Acri. } \\
\text { smart }\end{array}$ & $\begin{array}{c}\text { One- } \\
\text { piece }\end{array}$ & Acrylate & Flat & -0.26 & 0 & 6.0 \\
\hline IQ & 30 & Alcon & SN60WF & $\begin{array}{c}\text { One- } \\
\text { piece }\end{array}$ & Acrylate & 2 & -0.20 & 0 & 6.0 \\
\hline $\begin{array}{l}\text { KS- } \\
\text { AiN }\end{array}$ & 31 & $\begin{array}{c}\text { Japan Canon } \\
\text { Staar }\end{array}$ & KS-AiN & $\begin{array}{c}\text { Three- } \\
\text { piece }\end{array}$ & Silicone & 2 & -0.20 & 10 & 6.0 \\
\hline
\end{tabular}

which was perpendicular to line L1 and the pupillary axis (see Figure 1A). All measurements were performed by experienced ophthalmic technicians.

\section{Statistical Analysis}

SPSS 17.0 software was used for statistical analysis. Normality was estimated using the KolmogorovSmirnov test. The homogeneity of variance was evaluated using Levene's test. When a normal distribution and equal variance were expected, a one-way analysis of variance was performed to assess outcomes, while an independent samples $t$-test was used to compare two groups. Pearson's correlation analysis was used to evaluate the correlation between decentration or tilt and coma. A $p$-value of $<0.05$ was considered to be statistically significant.

\section{Results}

Comparison of IOL decentration, tilt, and coma after implantation of four different types of aspheric IOL 1 week and 1 year postoperatively

One week postoperatively, the mean IOL decentration in the AO, 36A, IQ, and KS-AiN groups was $0.109 \pm$ $0.027 \mathrm{~mm}, 0.128 \pm 0.036 \mathrm{~mm}, 0.155 \pm 0.048 \mathrm{~mm}$, and $0.185 \pm 0.025 \mathrm{~mm}$, respectively, which were statistically significantly different $(\mathrm{F}=1262.583, p=0.001$; Figure 2A). The mean IOL tilt in the AO, 36A, IQ, and KS-AiN groups was $2.105^{\circ} \pm 0.184^{\circ}, 2.284^{\circ} \pm 0.202^{\circ}, 2.564^{\circ} \pm$ $0.163^{\circ}, 2.922^{\circ} \pm 0.172^{\circ}$, respectively $(\mathrm{F}=779.076, p=$ 0.001 ; Figure 2B). The mean coma in the AO, 36A, IQ, and KS-AiN groups was $0.048 \pm 0.012 \mu \mathrm{m}, 0.046 \pm 0.011$ $\mu \mathrm{m}, 0.086 \pm 0.009 \mu \mathrm{m}$, and $0.117 \pm 0.015 \mu \mathrm{m}$, respectively $(\mathrm{F}=220.268, p=0.001$; Figure $2 \mathrm{C})$.
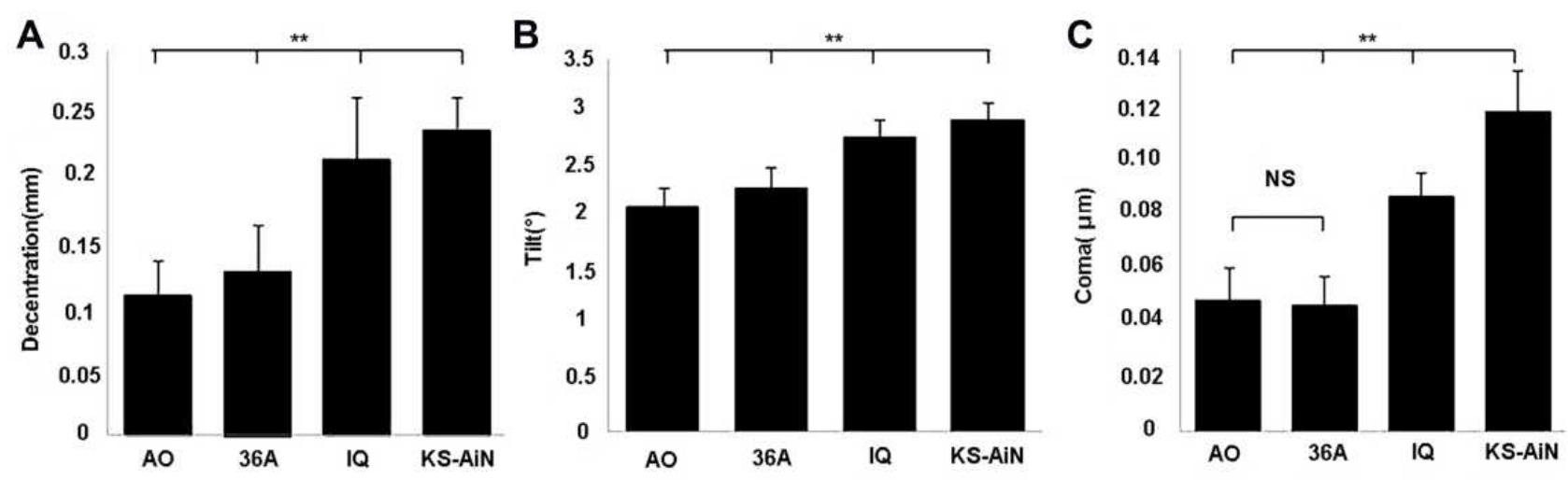

Figure 2 IOL decentration, tilt, and coma in the AO, 36A, IQ, and KS-AiN groups I week postoperatively. (A) Decentration. (B) Tilt. (C) Coma. (** $p<0.01$; NS $p>0.05$ ). 

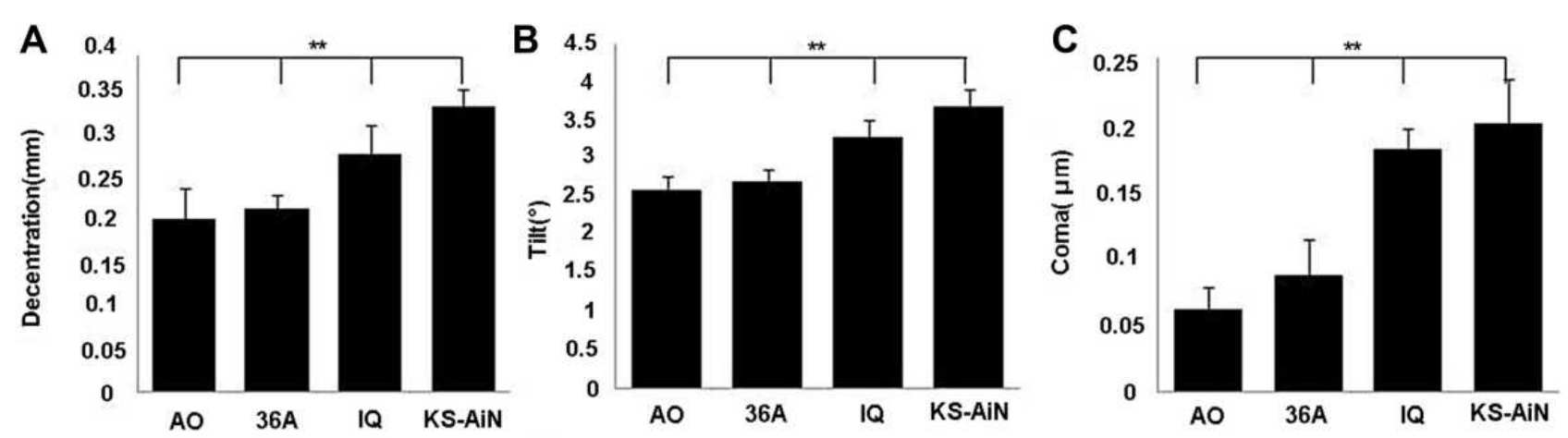

Figure $3 \mathrm{IOL}$ decentration, tilt, and coma in the AO, 36A, IQ, and KS-AiN groups I year postoperatively. (A) Decentration. (B) Tilt. (C) Coma. (** $p<0.0 \mathrm{I}$; NS $p>0.05$ ).
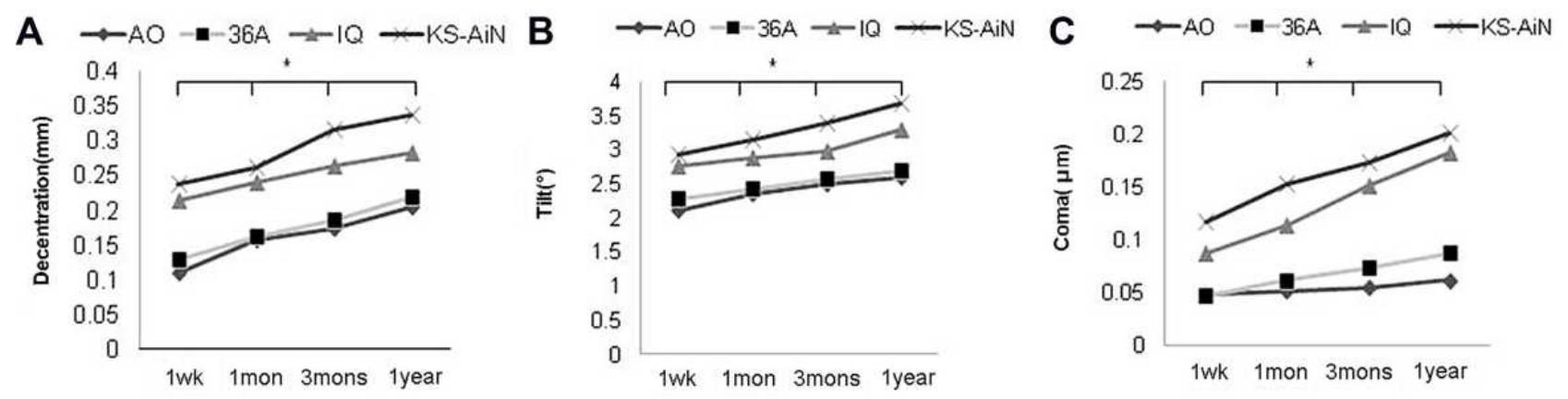

Figure $4 \mathrm{IOL}$ decentration, tilt, and coma in the AO, 36A, IQ, and KS-AiN groups at I week, I month, 3 months, and I year postoperatively. (A) Decentration. (B) Tilt. (C) Coma. $(* p<0.05$; NS $p>0.05)$.
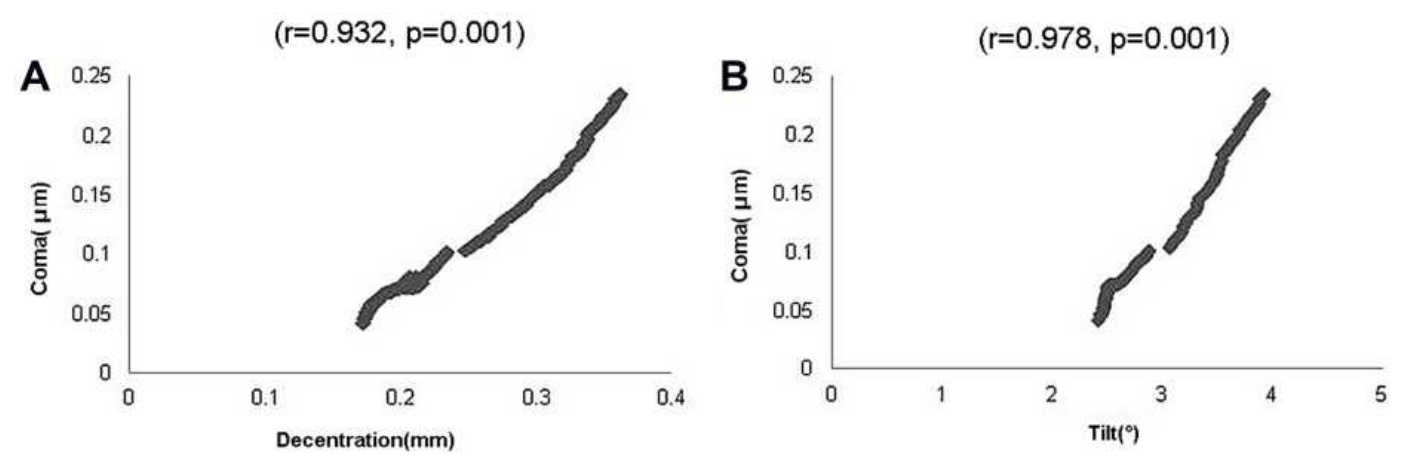

Figure 5 Correlation analysis between coma and decentration or tilt I year postoperatively. (A) Correlation analysis between decentration and coma. (B) Correlation analysis between tilt and coma.

When comparing IOL decentration and tilt in four groups, statistically significant differences were observed $(p<0.01)$. Coma was statistically significantly different $(p<0.01)$ between the IQ and KS-AiN groups, but was not significantly different between the AO and 36A groups $(p>0.05$; Figure 2A-C).

One year postoperatively, the mean IOL decentration in the AO, 36A, IQ, and KS-AiN groups was $0.205 \pm$ $0.038 \mathrm{~mm}, 0.218 \pm 0.016 \mathrm{~mm}, 0.282 \pm 0.035 \mathrm{~mm}$, and
$0.338 \pm 0.022 \mathrm{~mm}$, respectively $(\mathrm{F}=82.184, p=0.001$; Figure $3 \mathrm{~A}$ ). The mean IOL tilt in the AO, 36A, IQ, and KS-AiN groups was $2.586^{\circ} \pm 0.187^{\circ}, 2.692^{\circ} \pm 0.182^{\circ}$, $3.286^{\circ} \pm 0.228^{\circ}$, and $3.683^{\circ} \pm 0.234^{\circ}$, respectively $(\mathrm{F}=$ $79.085, p=0.001$; Figure 3B). The mean coma in the $\mathrm{AO}, 36 \mathrm{~A}$, IQ, and KS-AiN groups was $0.061 \pm 0.018$ $\mu \mathrm{m}, 0.087 \pm 0.028 \mu \mathrm{m}, 0.182 \pm 0.016 \mu \mathrm{m}$, and $0.201 \pm$ $0.034 \mu \mathrm{m}$, respectively $(\mathrm{F}=101.286, p=0.001$; Figure 3C). 
When comparing the 1-week and 1-year time points, IOL decentration, tilt, and coma were statistically significantly different $(p<0.01$; Figure $3 \mathrm{~A}-\mathrm{C})$.

Comparison of IOL decentration, tilt, and coma after implantation of four different types of aspheric IOL 1 week, 1 month, 3 months, and 1 year postoperatively

One week, 1 month, 3 months, and 1 year postoperatively, IOL decentration, tilt, and coma with each type of aspheric IOL were statistically significantly different $(p<0.05$; Figure 4A-C). IOL decentration, tilt, and coma increased in each four different types of aspheric IOL during the postoperative period from 1 week to 1 year.

Correlation analysis between IOL decentration or tilt and coma 1 year postoperatively

According to Pearson's correlation analysis, there was a positive linear correlation between IOL decentration and coma 1 year postoperatively $(r=0.932, p=0.001$; Figure $5 \mathrm{~A})$. There was also a positive linear correlation between IOL tilt and coma 1 year postoperatively $(\mathrm{r}=0.978, p=$ 0.001; Figure 5B).

\section{Discussion}

The extensive clinical application of aspheric IOLs has demonstrated obvious advantages over spherical IOLs in reducing postoperative wavefront aberrations and improving visual quality. Therefore, aspheric IOLs are still the main type of IOL applied in patients with cataracts. However, due to their different haptic designs, optical materials, and biocompatibilities, different types of aspheric IOLs have different positional stabilities, which may affect their ability to change wavefront aberrations. After implantation in the capsular bag, IOLs undergo compression and capsular contraction. In this study, it was observed that acrylic aspheric IOLs with more haptics exhibited significantly less capsular contraction in the lens and enhanced the attachment of the posterior surface of the IOL optic region to the posterior capsule. Therefore, IOLs may have fewer ocular coma-like aberrations owing to their better positional stability.

Phacoemulsification combined with intraocular lens inthe-bag implantation is the mainstream approach to cataract surgery. With ongoing improvements in surgery, ever increasing postoperative visual quality is required. Loss of visual acuity, glare, and halos, which can be caused by decentration and tilt of IOLs, are receiving increasing attention. Ocular coma-like aberrations caused by decentration and tilt of IOLs can result in myopic shift and oblique astigmatism, which are difficult to correct with regular glasses. ${ }^{12-14}$ Aspheric IOLs can significantly improve visual function in humans and have good optical performance and application prospects. However, coma, which results from serious decentration and tilt of aspheric IOLs, may cause an even lower optical transfer function than when using spherical IOLs, especially in high frequency sections. ${ }^{15}$ Thus, to guide personalized clinical treatment, it is important to evaluate decentration, tilt, and the corresponding changes in coma of various types of aspheric IOLs.

A number of factors can lead to decentration and tilt in IOLs including non-in-the-bag IOL implantation, inconsistent diameter between the capsular bag and the IOL (including the loop), and capsular bag tear. ${ }^{7}$ Continuous circular capsulorhexis is currently regarded as the best way to reduce radial tears and keep the IOL centered. In this study, for the purpose of eliminating all other factors that could affect IOL stability, aside from the IOL itself, only patients with agerelated cataracts were selected, and all surgeries were performed by the same surgeon with in-the-bag IOL implantation and no posterior capsular rupture. Cortex and lens epithelial cells were completely removed to prevent residual cell proliferation and excessive fibrous scarring, which can lead to capsular bag contraction and off-centering of the optical section of the IOL.

In addition, in cataract extraction and IOL implantation, the capsular bag starts to shrink, and it usually takes two to three months to fix the IOL completely. ${ }^{16}$ The IOL does not fully connect with the peripheral capsule during the first week after implantation, ${ }^{17}$ and the pressure from the contraction and deformation of the capsular bag during this accommodation period can asymmetrically affect the decentration and tilt of the IOL. ${ }^{18}$ The decentration and tilt differ between types of IOLs. In this study, IOL decentration, tilt, and coma in the AO, 36A, IQ, and KS-AiN implantation groups were statistically significantly different at one week and one year postoperatively. In the $\mathrm{AO}$ and 36A groups, the values were low, in the IQ group the value was medium, and in the KSAiN group the value was high. There are a number of possible reasons for these differences. First, due to the compressibility of the loops in one-piece IOLs, the pressure from capsular bag contraction and capsular deformation during accommodation mainly affects the loops rather than the optical section of the IOL, causing less decentration and tilt when compared with three-piece IOLs. ${ }^{19}$ When compared with two-point fixed IOLs, the ability of four-point fixed 
IOLs to disperse the pressure from one point to another may reduce decentration and tilt. In this study, the similar IOL decentration, tilt, and coma between the $\mathrm{AO}$ and 36A groups may be due to the similar flat four-point fixed design of these IOLs. $^{20}$

No previous studies have shown a relationship between IOL stability and time. This study compared different time points (one week, one month, three months, and one year postoperatively) with regard to IOL decentration, tilt, and coma in the four groups, and statistically significant differences were observed. The values increased at different levels over time. The values were low in the $\mathrm{AO}$ and 36A groups, medium in the IQ group, and high in the KSAiN group. The reason for these variations may be closely related to adhesion between the IOL and the capsule. Adhesion is weaker in silicone IOLs when compared with acrylic IOLs. ${ }^{21}$ As a result, decentration and tilt are more obvious in silicone than acrylic IOLs under pressure caused by continuous capsular bag contraction over time.

The correlation analysis showed a positive linear correlation between IOL decentration or tilt and coma, which indicated that coma could reflect the position of the IOL in the capsule. These results are consistent with Oshika's study on the correlation between the position of the IOL and coma. ${ }^{11}$ In addition, based on the correlation index in this study, IOL tilt may better reflect coma than decentration. In a previous study on decentration and tilt after IOL implantation, Hayashi et $\mathrm{al}^{22}$ showed that the average IOL decentration was $0.21-0.26 \mathrm{~mm}$, and the average tilt was $1.40^{\circ} \sim 1.77^{\circ}$. Baumeister et $\mathrm{al}^{23}$ observed an average IOL decentration of $0.23 \sim 0.29 \mathrm{~mm}$ and an average tilt of $2.32^{\circ}$ $\sim 3.26^{\circ}$ using the Scheimpflug system. These results are supported by the present study. The positive linear correlation between IOL decentration or tilt and coma strongly supports the influence of IOL stability on coma. Aspheric intraocular lens implantation can significantly reduce spherical aberration, contrast sensitivity in low spatial frequency is greater than in spherical intraocular lenses, and contrast sensitivity in glare and non-glare conditions is greater than in the normal people.

When comparing the $\mathrm{AO}$ and 36A groups one week and one year postoperatively, statistically significant differences in IOL decentration and tilt were observed, while no significant differences were observed in coma one week postoperatively. However, as IOL decentration and tilt increased, there were statistically significant differences in IOL decentration, tilt, and coma one year postoperatively. The observation that coma varied significantly only when decentration was greater than $0.2 \mathrm{~mm}$ and tilt was greater than $2.8^{\circ}$ demonstrates that coma can be influenced dramatically when variations in IOL stability reach a certain level but might not be affected by slight changes in IOL stability.

In conclusion, our data show that good positional stability fully utilizes the advantages of aspheric IOLs and improves the visual quality of patients. Based on differences in IOL decentration, tilt, and coma after implantation of four types of aspheric IOLs, it can be concluded that one-piece multi-point fixed acrylic IOLs have better stability when compared with three-piece two-point fixed silicone IOLs. IOL decentration, tilt, and coma increase gradually from one week to one year postoperatively. Ocular high-order wavefront aberrations, especially ocular coma-like aberrations, are influenced by IOL stability but not by slight changes in IOL decentration and tilt.

\section{Ethic Number}

EC-2019-KS-062.

\section{Acknowledgments}

We would like to acknowledge the hard and dedicated work of all the staff that implemented the intervention and evaluation components of the study.

\section{Funding}

Youth Project of National Natural Science Foundation of China (No. 81600717); Natural Science Foundation of Liaoning Province, China (No. 201602851).

\section{Disclosure}

The authors report no conflicts of interest in this work.

\section{References}

1. Yao K. Surgeries in complicated cases of cataract. Peking Beijing Sci Technol Press. 2004;252.

2. Lu YY, Yang N, Li XD, Kong J. Analysis on shift and rotation of intraocular lens after phakic collamer lens implantation. Int J Ophthalmol. 2016;16:848-851.

3. Fang YW, Lu Y, Wang L. Decentration and tilt of foldable intraocular lenses in the lens capsule. Chin $J$ Optometry Ophthalmol. 2008; 10:252-258

4. Zhang F, Zhang J, Li W, et al. Correlative comparison of three ocular axes to tilt and decentration of intraocular lens and their effects on visual acuity. Ophthalmic Res. 2020;63:165-173. doi:10.1159/ 000504716

5. Yasuaki I, Naoyuki M, Toshihide I, Hiroshi N, Kazuyuki E. Impact of vitrectomy and air tamponade on aspheric intraocular lens tilt and decentration and ocular higher-order aberrations: phacovitrectomy Versus Cataract Surgery.[J]. Jpn J Ophthalmol. 2020;64:359-366. doi:10.1007/s10384-020-00737-0 
6. Li CL, Cui H, Li ZR, et al. Effect of inclination and eccentricity of intraocular lens on optical imaging quality detected by wavefront aberration system. Int J Ophthalmol. 2019;19:2071-2075.

7. Zhu HF, Fang ZL, Liu YJ. Effect of intraocular lens decentration and tilt on vision performance of human eye. Acta Photonica Sin. 2008;37:141-144.

8. Piers PA, Weeber HA, Artal P, Norrby S. Theoretical comparison of aberration-correcting customized and aspheric intraocular lenses. J Refract Surg. 2007;23:374-384. doi:10.3928/1081-597X-2007040110

9. Lawu T, Mukai K, Matsushima H, Senoo T. Effects of decentration and tilt on the optical performance of 6 aspheric intraocular lens designs in a model eye. J Cataract Refract Surg. 2019;45:662-668. doi:10.1016/j.jcrs.2018.10.049

10. Lin J, Liao X, Lan CJ, Tan QQ, Wen BW, Tian J. Visual quality research on a custom selection of aspheric intraocular lenses in cataract patients. $J$ Otolaryngol Ophthalmol Shandong Univ. 2019;33:109-144.

11. Oshika T, Sugita G, Miyata K, et al. Influence of tilt and decentration of scleral-sutured intraocular lens on ocular higher-order wavefront aberration. $\mathrm{Br} J$ Ophthalmol. 2007;91:185-188. doi:10.1136/ bjo.2006.099945

12. Huseynova T, Mita M, Corpuz CC, Sotoyama Y, Tomita M. Evaluating the different laser fragmentation patterns used in laser cataract surgeries in terms of effective phacoemulsification time and power. Clin Ophthalmol. 2015;6(9):2067-2071.

13. Taketani F, Matuura T, Yukawa E, Hara Y. Influence of intraocular lens tilt and on wavefront aberrations. J Cataract Refract Surg. 2004;30:2158-2162. doi:10.1016/j.jcrs.2004.02.072

14. Oshika T, Kawana K, Hiraoka T, Kaji Y, Kiuchi T. Ocular higher-order wavefront aberration caused by major tilting of intraocular lens. Am J Ophthalmol. 2005;140:744-746. doi:10.1016/j. ajo.2005.04.026

15. De Castro A, Rosales P, Mareos S. Tilt and decentration of intraocular lenses in vivo from Purkinje and Scheimpflug imaging validation study. J Cataract Refract Surg. 2007;33:418-429. doi:10.1016/j. jers.2006.10.054
16. He SZ. Diseases of the Lens. Peking People's Med Publ House. 2003;325.

17. Modesti M, Pasqualitto G, Appolloni R, Pecorella I, Sourdille P. Preoperative and postoperative size and movements of the lens capsular bag: ultra-sound biomicroscopy analysis. J Cataract Refract Surg. 2011;37:1775-1784. doi:10.1016/j.jcrs.2011.04.035

18. Shi D, Zhang JS. Impact of different intraocular lens design on the wave-front aberration of pseudophakic eyes. Int $J$ Ophthalmol. 2008; 18:71-73.

19. Xing XJ, Tang X, Song H, Li WW. Comparison of tilt and decentration of four different kinds of aspheric intraocular lenses implantation. Chin J Ophthalmol. 2010;46:332-336.

20. Song H, Yuan X, Tang X. Effects of intraocular lenses with different diopters on chromatic aberrations in human eye models. $B M C$ Ophthalmol. 2016;16:9. doi:10.1186/s12886-016-0184-6

21. Kumar DA, Agarwal A, Packialakshmi S, Agarwal A. In vivo analysis of glued intraocular lens position with ultrasound biomicroscopy. J Cataract Refract Surg. 2013;39:1017-1022. doi:10.1016/j.jcrs.2013.01.039

22. Hayashi K, Hayashi H. Comparison of the stability of 1-piece and 3-piece acrylic intraocular lenses in the lens capsule. J Cataract Refract Surg. 2005;31:337-342.

23. Baumeister M, Neidhardt B, Strobel J, Kohnen T. Tilt and decentration of three-piece foldable high-refractive silicone and hydrophohic acrylic intraocular lenses with 6-mm optics in an intraindividual comparison. Am J Ophthalmol. 2005;140:1051-1058. doi:10.1016/j. ajo.2005.07.026
International Journal of General Medicine

\section{Publish your work in this journal}

The International Journal of General Medicine is an international, peer-reviewed open-access journal that focuses on general and internal medicine, pathogenesis, epidemiology, diagnosis, monitoring and treatment protocols. The journal is characterized by the rapid reporting of reviews, original research and clinical studies

\section{Dovepress}

across all disease areas. The manuscript management system is completely online and includes a very quick and fair peer-review system, which is all easy to use. Visit http://www.dovepress.com/ testimonials.php to read real quotes from published authors. 